\title{
Demonstration of spin-orbit interaction of a photon in a multimode rectilinear optical fiber
}

\section{Nataliya Kundikova}

Nataliya D. Kundikova, "Demonstration of spin-orbit interaction of a photon in a multimode rectilinear optical fiber," Proc. SPIE 9666, 11th Education and Training in Optics and Photonics Conference, 96661S (5 June 2009); doi: $10.1117 / 12.2208087$

SPIE Event: Eleventh International Topical Meeting on Education and Training in Optics and Photonics, 2009, St. Asaph, United Kingdom 


\title{
Demonstration of spin-orbit interaction of a photon in a multimode rectilinear optical fiber
}

\author{
Nataliya D. Kundikova* \\ South Ural State University, Chelyabinsk, Russia \\ Institute of Electrophysics, Ural Branch of RAS, Ekaterinburg, Russia
}

\begin{abstract}
An experimental set up for demonstration and investigation of the spin-orbit interaction of a photon under polarized light propagation through a multimode rectilinear optical fiber is proposed. The influence of the trajectory on the light polarization can be observed under linear polarized skew ray's propagation. The angle of plane polarization rotation depends on the angle of incidence. The influence of the light polarization on the trajectory can be observed under circular polarized skew ray's propagation. The angle of the speckle pattern rotation under circular polarization sign change depends on the angle of incidence too.
\end{abstract}

Keywords: spin-orbit interaction of a photon, multimode optical fiber, light polarization.

\section{INTRODUCTION}

The spin-orbit interaction of a photon manifests itself in two effects. The first one is the influence of the trajectory on the light polarization. The second one is the influence of the polarization on the trajectory of light.

It has been shown ${ }^{1-4}$ that the plane of the light polarization rotates under the light propagation along the non-planar trajectory. If vectors tangential to this trajectory at the input and exit points are parallel, then the angle of rotation (in radians) is numerically equal to the solid angle W (in steradians) subtended by the trajectory tangential vector at the unit sphere ${ }^{2}$. The same result ${ }^{3}$ has been obtained on the basis of the adiabatic theorem (geometric phase) of M. Berry ${ }^{4}$. The change in the azimuth of linear polarized light has been observed experimentally under light propagation through a helical (coiled into a spiral) single mode fiber ${ }^{5}$. This effect has also been observed in a nonplanar Mach-Zender interferometer ${ }^{6}$.

The influence of the light polarization on the trajectory was demonstrated for the first time under total internal reflection. It was shown that a reflected ray suffers a longitudinal shift ${ }^{7,8}$. The value of the shift $L_{1}$ is on the order of the light wavelength and depends on the azimuth of linear polarized light. Experimental observation of this shift was made by Goos and Hanchen ${ }^{9,10}$. It has been shown that in addition to the longitudinal shift a transverse shift should be also observed and the direction (sign) of that shift depends on the sign of the circular polarized light ${ }^{11,12}$. The value of the transverse shift $L_{\mathrm{t}}$ is related to the longitudinal shift $L_{1}$ as follows: $L_{\mathrm{t}}=a L_{1}$, where $a<1$. The experimental observation of this effect was made in papers $^{13-15}$ under light propagation through a triangle prism.

It should be stressed that the longitudinal and transverse shifts of a ray under total internal reflection and the influence of the light trajectory on its polarization were not considered as the manifestation of the spin-orbit interaction of a photon. For the first time the influence of the trajectory on the light polarization and the influence of the polarization on the trajectory were considered as mutually inverse effects in $1990^{16}$. This consideration has led to the prediction of a new effect: rotation of the speckle pattern in a multimode optical fiber under the change of circular polarization from left-handed to right-handed. The effect is known as the optical Magnus effect and has been observed for the first time under light propagation through rectilinear multimode optical fiber ${ }^{17}$. In terms of quantum mechanics this effect has been regarded as an interaction between the orbital momentum of photon (photon trajectory) and its spin (polarization) ${ }^{17}$.

Computer modeling of circular polarized light propagation through a multimode optical fiber and thorough experimental investigation of the optical Magnus effect has been carried out in paper $^{18}$. The influence of the trajectory on the

\footnotetext{
*E-mail: knd@susu.ac.ru
} 
light polarization and the influence of the polarization on the trajectory in the frame of the unified Hamiltonian were considered in paper ${ }^{19}$.

The name of optical Magnus effect is connected with the following analogy ${ }^{18}$. By looking at a rotating ping-pong ball falling down through the air, we can see that it is deflected from the vertical due to the Magnus effect. If we assume that a circularly polarized photon propagating in a waveguide is like our rotating ball, we can expect it's deviation from the initial trajectory.

Influence of the trajectory on the light polarization was observed in a single mode optical fiber coiled into a spiral ${ }^{5}$, and the optical Magnus effect, that is the influence of the light polarization on the beam trajectory, was observed under light propagation through a multimode rectilinear optical fiber ${ }^{17,18}$. If these two effects are mutually inverse, they should be observed under identical conditions, i.e. in the same optical fiber.
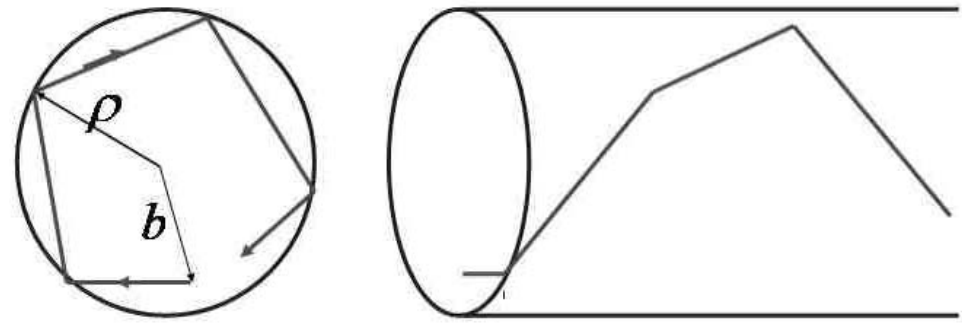

Fig. 1. Helical trajectory of "skew rays" in a rectilinear multimode optical fiber

Let us consider "skew rays" propagation through a rectilinear multimode optical fiber (Fig.1). The "skew rays" can be exited in the multimode optical fiber if light is fallen at any angle $b$ to the fiber axis. The rays form a helical trajectory and we can expect a rotation of the polarization plane after light transmission through the fiber ${ }^{20}$. The notion of a ray is taken from geometric optics and cannot be applied to the light propagation through the multimode optical fiber. The mode approach, however, is suitable, but some results can be obtained in the frame of geometrical optics. The interference of the different fiber modes produces a speckle pattern at a screen after the transmission of the light through the fiber. The speckle pattern looks like a narrow ring (Fig. 2), and the optical Magnus effect can be observed under change of the circular polarization sign ${ }^{21}$.

Here we are going to show how it is possible to demonstrate the influence of the trajectory on the light polarization and the influence of the polarization on the trajectory under light propagation through a rectilinear multimode optical fiber.

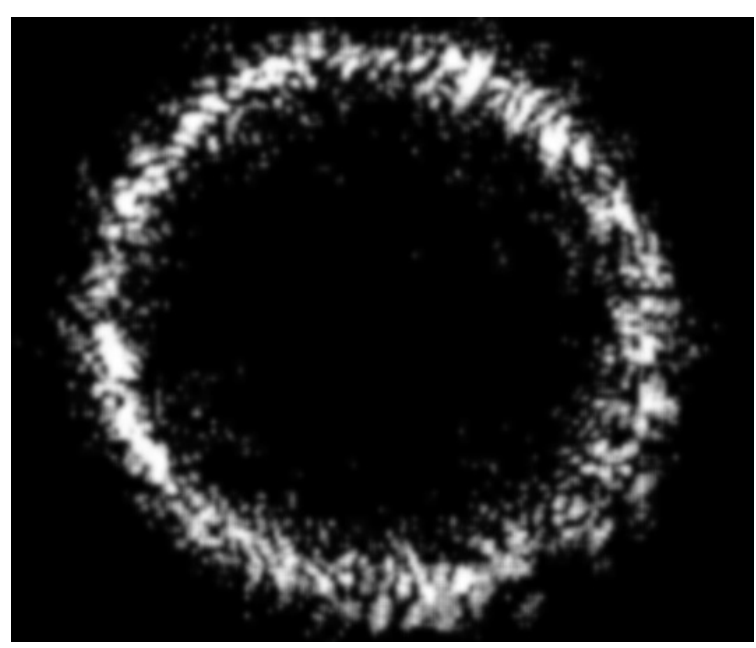

a

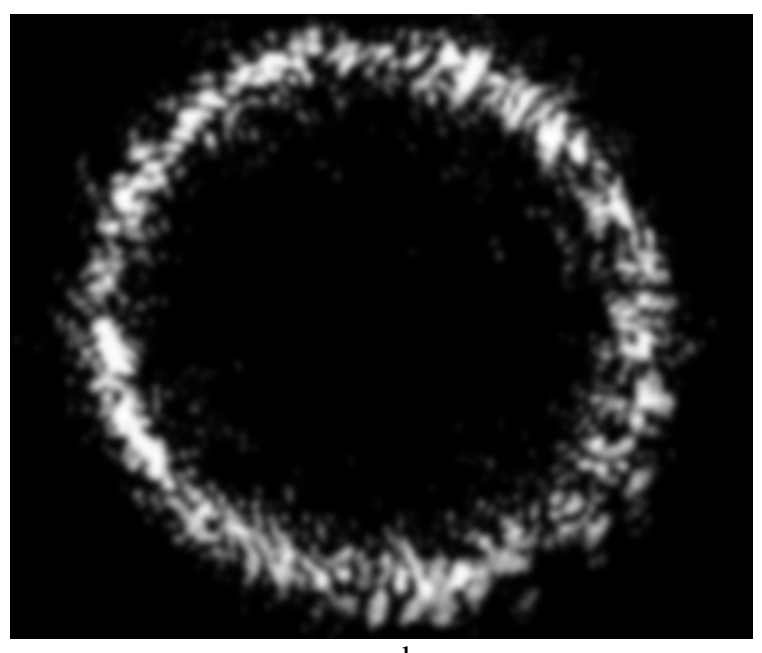

b

Fig.2. Speckle pattern of the light transmitted through multimode optical fiber under none zero angle of the light incidence at the fiber input for left- (a) and right- (b) handed circular polarized light. 


\section{LIGHT PROPAGATION THROUGH A MULTIMODE FIBER WITH A STEP-LIKE REFRACTIVE} INDEX PROFILE

Let us consider the propagation of the circular polarized light through an axially symmetrical fiber with a step-like refractive index profile $n(r)$ :

$$
\begin{array}{ll}
n(r)=n_{\mathrm{co}}, & r / \rho<1, \\
n(r)=n_{\mathrm{cl}}, & r / \rho>1 .
\end{array}
$$

Here $(x ; y)=\mathbf{r}$ are the transverse coordinates, $r=|\mathbf{r}|, r$ is the radius of the fiber core; $n_{\mathrm{co}}$ and $n_{\mathrm{cl}}$ are the refractive indices of the fiber core and the cladding respectively.

The polarization is not coupled to the propagation of the beam in the zero paraxial approximation (approximation of weakly guided waveguide), corresponding to the scalar parabolic equation. More accurately, the spatial structure of the field and its polarization are coupled due to the inhomogeneity of the refractive index. Axial symmetry of the fiber allows ${ }^{22}$ these modes to be taken in the following form:

$$
\begin{aligned}
\mathbf{e}_{m, N}^{(1)}(r, \varphi) & =\left[\cos (m \varphi) \mathbf{e}_{x}-\sin (m \varphi) \mathbf{e}_{y}\right] \cdot F_{|m|, N}(r), \\
\mathbf{e}_{m, N}^{(2)}(r, \varphi) & =\left[\cos (m \varphi) \mathbf{e}_{x}+\sin (m \varphi) \mathbf{e}_{y}\right] \cdot F_{|m|, N}(r), \\
\mathbf{e}_{m, N}^{(3)}(r, \varphi) & =\left[\sin (m \varphi) \mathbf{e}_{x}+\cos (m \varphi) \mathbf{e}_{y}\right] \cdot F_{|m|, N}(r), \\
\mathbf{e}_{m, N}^{(4)}(r, \varphi) & =\left[\sin (m \varphi) \mathbf{e}_{x}-\cos (m \varphi) \mathbf{e}_{y}\right] \cdot F_{|m|, N}(r) .
\end{aligned}
$$

Here $\mathbf{e}_{x}$ and $\mathbf{e}_{y}$ are unit vectors, $x=r \cos \varphi, y=r \sin \varphi, m=0, \pm 2, \pm 3, \pm 4, \ldots$ is the orbital momentum, and $N=1,2,3, \ldots$ is the radial quantum number, $F_{|m|, N}(r)$ is the radial function:

$$
\begin{array}{ll}
F_{|m|, N}(r)=J_{|m|}\left(U_{N} r\right), & r / \rho<1, \\
F_{|m|, N}(r)=K_{|m|}\left(W_{N} r\right), & r / \rho>1,
\end{array}
$$

where $J_{|m|}\left(U_{N} r\right)$ is Bessel function and $K_{|m|}\left(W_{N} r\right)$ is MacDonald function. The values $U_{N}$ and $W_{N}$ for given $m$ are determined from the equation:

$$
U_{N} \frac{J_{m+1}\left(U_{N}\right)}{J_{m}\left(U_{N}\right)}=W_{N} \frac{K_{m+1}\left(U_{N}\right)}{K_{m}\left(U_{N}\right)},
$$

where $V^{2}=W_{N}^{2}+U_{N}^{2} \quad, \quad V=\rho k n_{\mathrm{co}}(2 \Delta)^{1 / 2} \quad$ is a dimensionless parameter $\quad(V \square 1$ for a multimode fiber), $\Delta=\left(n_{\mathrm{co}}^{2}-n_{\mathrm{cl}}^{2}\right) / 2 n_{\mathrm{co}}^{2} \approx\left(n_{\mathrm{co}}-n_{\mathrm{cl}}\right) / n_{\mathrm{co}}=\Delta n / n_{\mathrm{co}} \square 1, k=2 \pi / \lambda$, and $\lambda$ is the wavelength of light in the air.

Any linear combinations of modes $\mathbf{e}_{m, N}^{(1)}(r, \varphi)$ and $\mathbf{e}_{m, N}^{(3)}(r, \varphi)$ for all values of $m$ and any linear combinations of modes $\mathbf{e}_{m, N}^{(2)}(r, \varphi)$ and $\mathbf{e}_{m, N}^{(4)}(r, \varphi)$ for $m \neq 1$ are also eigenmodes. It is easily to show, that a combination of modes $\mathbf{e}_{m, N}^{(1)}(r, \varphi) \pm i \mathbf{e}_{m, N}^{(3)}(r, \varphi)$ and $\mathbf{e}_{m, N}^{(2)}(r, \varphi) \pm i \mathbf{e}_{m, N}^{(4)}(r, \varphi)(i=\sqrt{-1})$ are homogeneously polarized eigenmodes with circular polarization:

$$
\begin{aligned}
& \mathbf{e}_{+, m, N}^{+}(r, \varphi)=\mathbf{e}_{m, N}^{(1)}(r, \varphi)+i \mathbf{e}_{m, N}^{(3)}(r, \varphi)=\left(\mathbf{e}_{x}+i \mathbf{e}_{y}\right) \cdot \exp (i m \varphi) \cdot F_{|m|, N}(r), \\
& \mathbf{e}_{-, m, N}^{-}(r, \varphi)=\mathbf{e}_{m, N}^{(1)}(r, \varphi)-i \mathbf{e}_{m, N}^{(3)}(r, \varphi)=\left(\mathbf{e}_{x}-i \mathbf{e}_{y}\right) \cdot \exp (-i m \varphi) \cdot F_{|m|, N}(r), \\
& \mathbf{e}_{+, m, N}^{-}(r, \varphi)=\mathbf{e}_{m, N}^{(2)}(r, \varphi)+i \mathbf{e}_{m, N}^{(4)}(r, \varphi)=\left(\mathbf{e}_{x}-i \mathbf{e}_{y}\right) \cdot \exp (i m \varphi) \cdot F_{|m|, N}(r), \\
& \mathbf{e}_{-, m, N}^{+}(r, \varphi)=\mathbf{e}_{m, N}^{(2)}(r, \varphi)-i \mathbf{e}_{m, N}^{(4)}(r, \varphi)=\left(\mathbf{e}_{x}+i \mathbf{e}_{y}\right) \cdot \exp (-i m \varphi) \cdot F_{|m|, N}(r) .
\end{aligned}
$$

Let us consider now the important specific case in which we illuminate the fiber with right- and left-handed circular polarization in turn, but with strictly the same modal distribution $C_{ \pm, m, N}$ at the input. It is interesting to compare the field 
distributions for right- and left-handed circular polarization in some cross-section $z$. The field at the fiber input has the following distribution:

$$
\mathbf{E}^{ \pm}(r, \varphi, z=0)=\left(\mathbf{e}_{x} \pm i \mathbf{e}_{y}\right) \cdot\left[\sum_{m} \sum_{N} C_{-, m, N} e^{-i m \varphi} F_{|m|, N}(r)+\sum_{m} \sum_{N} C_{+, m, N} e^{i m \varphi} F_{|m|, N}(r)\right] .
$$

In the case under consideration it is possible to take into consideration only modes with $m>1$. Really, in order to obtain ring like speckle patter we should to excite only modes with high values of $m$. In that particular case the circular polarization of the wave, right- or left-handed, is conserved during the propagation and we obtain the following field distribution at the fiber output:

$$
\begin{aligned}
& \mathbf{E}^{+}(r, \varphi, z)= \\
& \left(\mathbf{e}_{x}+i \mathbf{e}_{y}\right) \cdot\left\{\sum_{m>1} \sum_{N} C_{-, m, N} e^{-i m \varphi} F_{m, N}(r) \exp \left[i z\left(\beta_{m, N}+\delta \beta_{m, N}^{(2)}\right)\right]+\sum_{m>1} \sum_{N} C_{+, m, N} e^{i m \varphi} F_{m, N}(r) \exp \left[i z\left(\beta_{m, N}+\delta \beta_{m, N}^{(1)}\right)\right]\right\},
\end{aligned}
$$

$\mathbf{E}^{-}(r, \varphi, z)=$

$$
\left(\mathbf{e}_{x}-i \mathbf{e}_{y}\right) \cdot\left\{\sum_{m>1} \sum_{N} C_{+, m, N} e^{+i m \varphi} F_{m, N}(r) \exp \left[i z\left(\beta_{m, N}+\beta_{m, N}^{(2)}\right)\right]+\sum_{m>1} \sum_{N} C_{-, m, N} e^{-i m \varphi} F_{m, N}(r) \exp \left[i z\left(\beta_{m, N}+\delta \beta_{m, N}^{(1)}\right)\right]\right\} .
$$

Here $\beta_{m, N}$ are propagation constants, $\delta \beta_{m, N}^{(1)}$ and $\delta \beta_{m, N}^{(2)}$ are polarization propagation corrections:

$$
\begin{aligned}
& \delta \beta_{m, N}^{(1)}=\delta \beta_{m, N}^{(3)}=-\frac{(2 \Delta)^{3 / 2}}{2 \rho} \frac{W_{N} U_{N}^{2}}{V^{3}} \frac{K_{m}\left(W_{N}\right)}{K_{m-1}\left(W_{N}\right)}, \\
& \delta \beta_{m, N}^{(2)}=\delta \beta_{m, N}^{(4)}=-\frac{(2 \Delta)^{3 / 2}}{2 \rho} \frac{W_{N} U_{N}^{2}}{V^{3}} \frac{K_{m}\left(W_{N}\right)}{K_{m+1}\left(W_{N}\right)} .
\end{aligned}
$$

Eq. (1) and Eq. (2) can be used to calculate the field distribution of different sign of circular polarization at the fiber output. To simulate narrow beam propagation one should select the narrow range of values of $m$. To compare two field distribution and to extract the pure rotation from the whole change of the speckle pattern the correlation function should be calculated:

$$
\begin{aligned}
& K_{i j}(\psi, z)=\int I_{i}(r, \varphi, z) I_{j}(r, \varphi+\psi, z) d r d \varphi \\
& I_{i}(r, \varphi, z)=\left|E^{i}(r, \varphi+\psi, z)\right|^{2} \quad(i=+,-; \quad j=-,+)
\end{aligned}
$$

The angle $\psi$ is the angle of the speckle pattern rotation.

Detailed analytic investigation of the influence of the polarization on the light trajectory ${ }^{23}$ has shown that the peripheral parts of the speckle pattern are shifted by a larger angle compared with the central part. The angle of rotation of the speckle pattern depends on the angle $\beta$ between the fiber axis and the direction of propagation of the beam incident on the fiber input $\mathrm{end}^{23}$ :

$$
\psi=\frac{\lambda \beta^{2}}{\pi n_{\mathrm{co}}^{3} \rho^{2}} L
$$

Here $L$ is the fiber length. The peripheral parts of the speckle pattern correspond to the largest angles of ray entry into the fiber and the central parts of the speckle pattern correspond to the smallest angles of rays entry into the fiber, so the difference in propagating length can results in the different angles of speckle pattern rotation.

\section{THE ROTATION OF THE PLANE OF POLARIZATION UNDER LIGHT PROPAGATION THROUGH A RECTILINEAR MULTIMODE FIBER WITH A STEP-LIKE REFRACTIVE INDEX PROFILE}

Let us consider a skew ray with impact parameter $b$ (the distance between the point of the ray entry and the fiber axis) and the angle $a$ between the direction of a ray propagation $\mathbf{S}_{1}$ inside the fiber and the fiber axis $z$. 


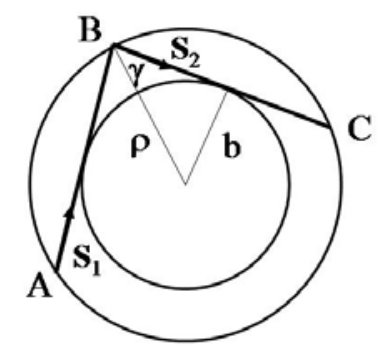

Fig. 2. Skew ray in a multimode optical fiber with step-like refraction index profile ( $\mathbf{r}$ space).

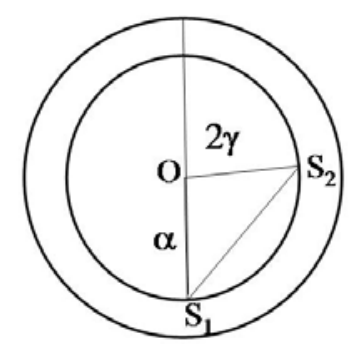

Fig. 3. Skew ray in a multimode optical fiber with step-like refraction index profile ( $\mathbf{S}$ space).

The angle $a$ can be regarded as small, i.e. $\tan a » \sin a » a$. As the ray is skew, the fiber diameter does not lay in the plane of incidence and impact parameter $b^{1} \quad 0$. The propagation of the ray in the section $A B$ (Fig. 2) in the coordinates space ( $\mathbf{r}$-space) corresponds to a point $\mathrm{S}_{1}$ (Fig. 3 ) on a unit sphere in the directions space ( $\mathbf{S}$ space). The point $\mathrm{S}_{1}$ lies on a circle of radius $a$ with it's center at the point $\mathrm{O}$. This point corresponds to the direction of the fiber axis $z$ in the coordinate space. After total internal reflection at a point B (Fig. 2) the ray assumes the direction $S_{2}$ corresponding to the point $S_{2}$ in the $\mathrm{S}$ space. This total internal reflection is localized at the point B in the coordinate spaces, but it corresponds to the line $\mathrm{S}_{1} \mathrm{~S}_{2}$ in the direction space. The point $\mathrm{S}_{2}$ still lies on a circle of radius $a$, but its azimuth changes by an angle $p-2 g$, where $\sin g=b / r$. As a result of a single total internal reflection the plane of polarization of the ray rotates by $y_{1}$, which is numerically equal to the area of the spherical triangle $\mathrm{OS}_{1} \mathrm{~S}_{2}$. This area of a spherical triangle $\mathrm{OS}_{1} \mathrm{~S}_{2}$ can be replaced with the area of plane triangle $\mathrm{OS}_{1} \mathrm{~S}_{2}$ in the approximation of small angles. Then

$$
y_{1}=\frac{1}{2} a^{2} \sin (2 g) \text {. }
$$

Taking into consideration that $\sin g=b / r$ we get:

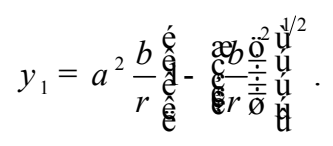

The ray passes the distance

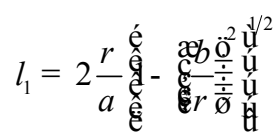

in $z$ - direction between two process of the total internal reflection, and the number of such elementary acts at the unite distance is $N=1 / l_{1}$ The angle of the plane polarization rotation at the length $L$ is

$$
y=N y_{1}=\frac{a^{3}}{2 r^{2}} b L
$$

If $\beta$ is the angle between the fiber axis and the direction of propagation of the beam incident on the fiber input end, then

$$
y=\frac{b^{3}}{2 n_{\mathrm{co}}^{3} r^{2}} b L .
$$

It is possible to derive Eq. (4) on the base of the wave theory ${ }^{23}$. 


\section{EXPERIMENTAL SET UP FOR DEMONSTRATION OF SPIN-ORBIT INTERACTION OF A PHOTON IN A MULTIMODE RECTILINEAR OPTICAL FIBER}

Figure 4 shows experimental set up for demonstration of spin-orbit interaction of a photon in a multimode rectilinear optical fiber. Linearly polarized light of a laser can be transformed into circular polarized light or linear polarized light with any azimuth by polarization system and was focused on the fiber input by a lens. Figure 5 shows optical scheme of the polarization system installed before the fiber input. It should be stressed that adjustable quarter wave plate, used in the polarization system, allows obtaining circular polarized light of very high quality ${ }^{24}$.

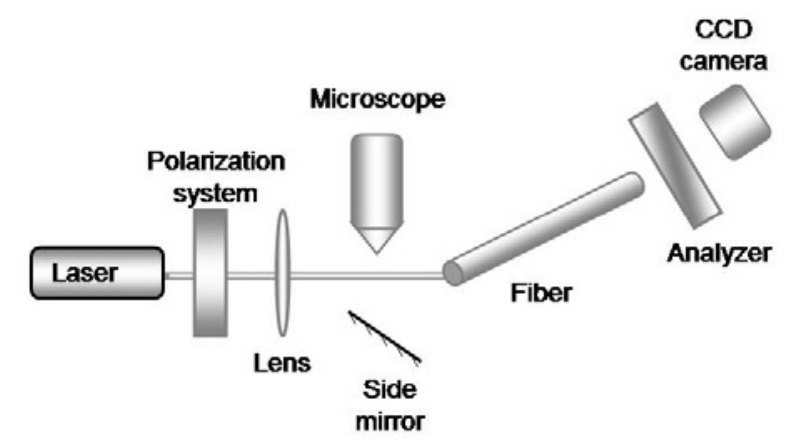

Fig. 4. Experimental set up.

\section{Polarizer}

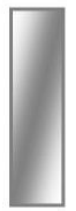

Polarizer

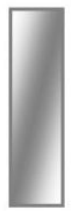

Adjustable quarter wave plate

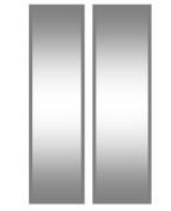

a
Polarizer
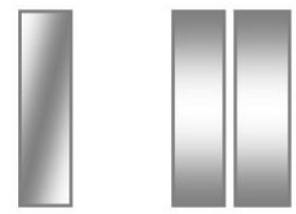

Adjustable quarter wave plate

b

Fig. 5. Polarization system for investigation of the plane polarization rotation (a) and investigation the optical Magnus effect (b).

The direction of the plane polarization rotation under the skew beam propagation coincides with the direction of the motion of $S_{1}$ points at the unit sphere (Fig. 3). If the beam is incident from the left side, we can change the direction of the plane polarization rotation illuminating the top or the bottom part of the input fiber end. A microscope is needed to control the illumination of the fiber input. The most convenient way to observe the fiber end is to use reflected light. Really we should deal with small angles of incidence, and a semitransparent mirror should be installed on the way of the linear polarized beam. Unfortunately linear polarized light transmitted through that mirror suffers depolarization. As a result the depolarized light is incident at the fiber input end and it can lead to fake effects. It is possible to solved this problem in the following way. It was found that intensity of the light focused at the fiber input is sufficient in order to observe the light scattered by micro inhomogeneities of the fiber end. So it is possible to observe the scattered light under the microscope by means of a side metal mirror. It allows controlling the illumination of the fiber input without disturbing the beam polarization. 
As for a multimode optical fiber, a fiber with the following parameters was used in papers ${ }^{20,21}$. The length of the quartz fiber under investigation was $L=7.5 \mathrm{~cm}$, the diameter of the fiber core was $2 \rho=100 \mu \mathrm{m}$, the difference $\Delta n$ between the refractive indices of the core $n_{\text {co }}$ and the refractive indices of the cladding $n_{\mathrm{cl}}$ was $\Delta n=n_{\mathrm{co}}-n_{\mathrm{cl}}=0.016$. The value of $\Delta n$ defines the maximum angle of incidence $\beta_{\max }$ when the meridional ray is confined to the fiber:

$$
\beta_{\text {max }}=\sqrt{2 n \Delta n} \approx 12.7^{\circ}
$$

The angle of the input beam divergence $\theta_{0}$ in the air measured by the criterion $e^{-1}$ of the intensity at maximum was $\theta_{0}=0.5^{\circ}$, i.e. $\theta_{0} \square \beta_{\max }^{20,21}$. The value of the radius of the illuminated spot at the fiber input end $a_{0}$ calculated from $\theta_{0}$, was $a_{0}=\left(k \theta_{0}\right)^{-1} \approx 1.5 \mu \mathrm{m} \square \rho$, where $k=2 \pi / \lambda$. Really, it is possible to chose any multimode optical fiber, but the fiber length should be so that linear polarization does not change and the fiber diameter should be so that $2 \rho \square a_{0}$. The angle of the input beam divergence $\theta_{0}$ in the air and a lens should be chosen in such a way that the value of the diameter of the illuminated region at the fiber input end $a_{0} \square 2 \rho$.

As was mentioned above, the notion of a ray can not be applied to the case under consideration. As a result a speckle pattern in the form of a narrow ring is observed after light transmission through a multimode optical fiber. The radius of the ring allows calculating the angle $\beta$ of the beam incidence at the fiber input. To obtain more accurate results the external radius $R_{\text {ex }}$ and the internal radius $R_{\text {int }}$ of the ring should be measured, then:

$$
\beta=\frac{R_{\mathrm{ex}}+R_{\mathrm{int}}}{2 D},
$$

where $D$ is the distance between the output end of the fiber and the screen. The full illumination of the ring and the presence of a speckle pattern within it do demonstrate that the notion of a ray does not work. Using analyzer at the fiber output one can observe, that the azimuth of output linear polarized light $y_{\text {out }}$ does not coincide with the azimuth of input plane polarized light $y_{\text {in }}$. The angle $y_{\text {out }}$ can be determined by the rotation of the analyzer until the intensity of the light at the screen achieved it's minimum value. The angle of the plane polarization rotation should be determined as $y=y_{\text {in }}-y_{\text {out }}$. One can see that the value of $y$ does not depend on the angle $y_{\text {in }}$. The illumination of the fiber input should be controlled and compared with the direction of the plane polarization rotation. That direction should coincide with the direction predicted from the theory.

The same experimental set up can be used for the optical Magnus effect demonstration and investigation. In order to observe the optical Magnus effect the analyzer installed after the fiber should be removed. The polarization system (Fig. 5) allows changing the sign of circular polarization by rotating of the adjustable quarter wave plate.

\section{INVESTIGATION OF THE ROTATION OF THE POLARIZATION PLANE AND THE OPTICAL MAGNUS EFFECT}

The described experimental set up allows to investigate the dependence of the angle of linear polarization plane rotation and the angle of speckle pattern rotation on the angle of rays incidence $y(b)$ and $\psi(\beta)$. The procedure of the investigation is the following. Changing the angle of incidence $\beta$ one should determine the value of the angle by measuring the radii $R_{\text {ex }}$ and $R_{\text {int }}$ of the speckle pattern at the screen and using Eq. (5). It should be determined what part of the fiber input, top or bottom is illuminated. Using the analyzer and the first scheme of the polarization system one should change the azimuth of linear polarized light falling at the fiber input and to determine the angle of the linear polarization plane rotation.

Using the second scheme of the polarization system and removing the analyzer one can record the speckle pattern of left- handed and right- handed circular polarized light transmitted through the fiber. To determine the angle of the speckle pattern rotation the special software is needed.

To check the dependence of the direction linear polarization plane rotation on the sign of the ray trajectory helicity one should readjust experimental set up to illuminate another part of the fiber input end and repeat the investigation. It is possible to check that the optical Magnus effect does not depend on the sign of the ray trajectory helicity. 
Changing the value of the angle $\beta$ the experimental dependencies $y(b)$ and $\psi(\beta)$ can be obtained. The experimental points should lie at the theoretical curves predicted by Eq. (4) and Eq. (3).

In conclusion, we have proposed the experimental set up which allows to demonstrate spin-orbit interaction of a photon under the same condition in the same fiber and to investigate the dependencies $y(b)$ and $\psi(\beta)$.

\section{REFERENCES}

[1] Rytov S. M., "About transition from wave to geometrical optics” Dokl. Akad. Nayk., 18, 2 (1938).

[2] Vladimirsky V. V., "About rotation of a plane of polarization in the curved light beam", Dokl. Akad. Nayk., 21, 222 (1941).

[3] Chiao R. Y., Wu Y.-S., “Manifestation of Berry’s Topological Phase for the Photon”, Phys. Rev. Lett., 57, 933 (1986).

[4] Berry M. V., "Quantum phase factors accompanying adiabatic changes", Proc. Roy. Soc. A, 392, 45 (1984).

[5] Tomita A., Chiao R. Y., “Observation of Berry’s topological phase by use of an optical fiber”, Phys. Rev. Lett., 57, 936 (1986).

[6] Chiao R. Y., Antaramian A., Ganga K. M., Jiao H., Wilkinson S. R., Nathel H., "Observation of a topological phase by means of a nonplanar Mach-Zehnder interferometer", Phys. Rev. Lett., 60, 1214 (1988).

[7] Picht J., "Beitrag zur Theorie der Totalreflexion", Ann. Physik, 3, 433 (1929).

[8] Picht J., "Die Energiestromung bei der Totalreflexion", Physik Z, 30, 905 (1929).

[9] Goos F., Hanchen H., "Ein neuer und Fundamentaler Versuch zurTotalreflexion”, Ann. Physik, 1, 333 (1947).

[10] Goos F., Hanchen H., "Neumessung des Strahlversetzungseffektes bei Totalreflexion", Ann. Physik, 5, 251 (1949).

[11]Fedorov F. I., "To the theory of total internal reflection", Dokl. Akad. Nayk, 105, 465 (1955).

[12] Kristofel N., "Total internal reflection and the effects connected to it", Proc. Tartu Univ., 42, 94 (1956).

[13] Imbert C., "Experimental proof of the photon's translational inertial spin effect”, Phys. Lett., 31A, 337 (1970).

[14] Imbert C., "Calculation and experimental proof of the transverse shift induced by total internal reflection of a circularly polarized light beam", Phys. Rev. D, 5, 787 (1972).

[15] Costa de Beauregard O., Imbert C., "Quantized longitudinal and transverse shifts associated with total internal reflection", Phys. Rev. Lett., 28, 1211 (1972).

[16]Zel'dovich B. Ya., Liberman V. S., "Rotation of the plane of a meridional beam in a graded-index waveguide due to the circular nature of the polarization", Sov. J. Quantum Electron. 20, 427 (1990).

[17] Dugin A. V., Zel'dovich B. Ya., Kundikova N. D., Liberman V. S., "Effect of circular polarization on the propagation of light through an optical fiber", JETP Lett., 53, 197 (1991).

[18] Dooghin A. V., Kundikova N. D., Liberman V. S., Zel'dovich B. Ya., “Optical Magnus effect”, Phys. Rev. A, 45, 8204 (1992).

[19] Liberman V. S., Zel'dovich B. Ya., "Spin-orbit interaction of a photon in an inhomogeneous medium”, Phys.Rev.A. 46, 5199 (1992).

[20]Zel'dovich B. Ya., Kundikova N. D., "Inhomogeneity of the optical Magnus effect”, Quantum Electron., 25, 172 (1995).

[21]Zel'dovich B. Ya., Kataevskaya I. V., Kundikova N. D., "Inhomogeneity of the optical Magnus effect", Quantum Electron., 26, 87 (1996).

[22] Snyder A. W., Love J. D., Optical Waveguide Theory, Chapman and Hall, London, New York (1983).

[23] Liberman V. S., Zel'dovich B. Ya., "Spin-orbit polarization effects in isotropic multimode fibres", Pure Appl. Opt., 2, 367 (1993).

[24] Goltser I. V., Darsht M. Ya., Kundikova N. D., Zel'dovich B. Ya., “An adjustable quarter-wave plate”, Optics Communications, 97, 291 (1993). 